Texture of Crystalline Solids. 1979, Vol. 3, pp. 231-243

0309-7951/79/0304-0231\$04.50/0

() 1979 Gordon and Breach Science Publishers, Inc.

Printed in Scotland

\title{
AN ANALYSIS OF ELASTIC ANISOTROPY AND MICROYIELDING IN POLYCRYSTALLINE MOLYBDENUM TZM
}

\author{
A. KUMAR \\ Metallurgy Division, Bhabha Atomic Research Centre, \\ Trombay, India \\ and \\ W. B. HUTCHINSON \\ Department of Metallurgy \& Materials, University of \\ Aston, Birmingham, England
}

\begin{abstract}
Samples of molybdenum TZM alloy in sheet form have been investigated in the warm-worked condition and after recrystallisation heat treatments to produce a range of different grain sizes. Orientation distributions were calculated from measured pole figure data and specimens were cut at various angles around the sheet to determine the planar variations in Young's modulus and microyield stress. Elastic moduli have been calculated for the materials on the basis of several different models using the preferred orientation data. Excellent agreement is found between the measured moduli and those calculated according to the Eshelby-Kröner-Kneer model. Variations in stress level from grain to grain were also calculated according to this model and used to predict the initiation of plastic flow, i.e. the microyield point. These predictions also agree closely with measured values. Variation of grain size did not have a major influence on elasticity or microyielding.
\end{abstract}

\section{INTRODUCTION}

Several attempts have been made to model the elastic behaviour of polycrystalline aggregates in terms of constituent single crystal behaviour. The initial models proposed by Voigt $^{1}$ and Reuss ${ }^{2}$ invoked the concepts of constant strain and stress states and yielded upper and lower bounds, respectively, of the polycrystalline elastic modulus. A refined set of bounds was obtained by Hashin et al. ${ }^{3}$ using variational principles. All these methods introduce simplifications regarding the mechanics of the problem. In real materials the interactions between differently oriented grains cause local perturbations in the stress and strain fields so that neither 
of these properties is constant throughout the body. The first successful attempt to meet these requirements was provided by Eshelby ${ }^{4}$ who showed that the strains inside an ellipsoidal isotropic inclusion, embedded in a homogeneous different isotropic matrix, are uniform. Kröner ${ }^{5}$ extended this approach to the problem of a homogeneous anisotropic spherical inclusion embedded in a homogeneous isotropic matrix. It was shown that for ellipsoidal inclusions with randomly oriented principal axes, the average stress experienced was equivalent to the stress for spherical inclusions. The non-uniform neighbourhood of a grain in a polycrystalline aggregate can be represented by a homogeneous matrix with the elastic constants of the polycrystal and that the grain can be replaced by a spherical inclusion. The problem of the anisotropic inclusion embedded in a homogeneous anisotropic matrix with differing elastic properties was solved by Kneer. ${ }^{6}$ Using such an approach, elastic constants of a polycrystalline aggregate with fibre texture were calculated. More recently, Morris ${ }^{7}$ has extended Kneer's approach to the case of an orthotropic polycrystalline aggregate as found, for example, in rolled sheets or plates. The sequence of hypothetical experiments performed by the above authors relevant to the present study are reviewed below. It should be noted that all of the more realistic models of an elastically deforming polycrystal imply that the stress tensor varies from grain to grain depending on orientation. The very early stages of plasticity, namely microyielding, should therefore be influenced by the effect of orientation on elastic behaviour of the material as well as by its control over the distribution of slip systems.

\section{The Inclusion Problem}

A spherical volume is removed from a homogeneous stress free medium having elastic constants, $C_{k l m n}$. The volume is subjected to a homogeneous shape change (stress free transformation), $e_{i j}^{T}$. Surface tractions are applied to elastically restore this to its original shape. It is replaced in the original hole and the layer of body force introduced at the interface is balanced by applying an equal and opposite distribution. At this stage, the total deformation of the volume is the constrained deformation, $e_{k}^{c}$. According to Eshelby, 4 the stress free and constrained deformations are proportional.

$$
e_{i j}^{T}=w_{i j k l} e_{k l}^{C}
$$

The stress in the volume can be represented as

$$
\sigma_{k 1}=c_{k 1 m n}\left(e_{m n}^{c}-e_{m n}^{T}\right)
$$

If an external elastic deformation $\bar{e}_{m n}$ is imposed on the matrix, the stress in the volume becomes

$$
\sigma_{k 1}=\left(C_{k 1 m n}-C_{k 1 t v} w_{t v m n}\right) e_{m n}^{c}+C_{k 1 m n} \bar{e}_{m n}
$$


This volume is removed and replaced by a single crystal inclusion which has been subjected to a net elastic deformation $\left(e_{m n}^{c}+\bar{e}_{m n}\right)$. The elastic constants $c_{k l m n}$ are chosen so that the original volume and the inclusion are equivalently stressed. The relations between the strain in the crystal (e) and the stress in the matrix $(\bar{\sigma})$, and between the stress in the single crys-

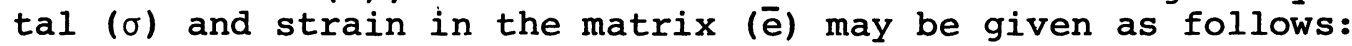

$$
\begin{aligned}
& e=\left(C^{-1}+u \ldots C^{-1}\right) \ldots \bar{\sigma} \\
& \sigma=(C+C \ldots(I-w) \ldots u) \ldots \bar{e}
\end{aligned}
$$

where $u=(c-c+c \ldots w)^{-1} \ldots(c-c)$, I is the identity matrix and (..) denotes tensor multiplication.

By direct analogy with dielectric theory, Kröner ${ }^{5}$ refers to the underlined term in Equation (5) as the "elastic polarisation" per unit volume. We can now turn to a polycrystalline material which is considered to be constituted from single crystal inclusions each of which is surrounded by an anisotropic but homogeneous medium. The properties of the medium or material as a body are found by correctly averaging the behaviour of these inclusions. In a continuous polycrystal, the average of the elastic polarisation over all grain orientations and grain shapes is zero. 6 For spherical inclusions in a polycrystalline medium, Equations (4) and (5) have a common solution, $\overline{\mathrm{u}}=0$. Thus for grain orientations $\Omega$ having a frequency of occurrence $V(\Omega)$ in a polycrystalline aggregate, the elastic constants are to be determined in such a way that the average elastic polarisation vanishes

$$
\frac{1}{v} \int u(\Omega) d v=0
$$

The aim of the present study is to solve numerically the polycrystalline elasticity problem for a range of accurately determined grain distribution functions and test the applicability of the theory by comparison with experimental observations. Calculations based on the theoretical development of Eshelby, Kröner and Kneer are referred to as the EKK values. In addition, the local stress-strain relations are used to evaluate the effect of elastic anisotropy on local yielding. This part of the study is also supported by experimental observations.

\section{EXPERIMENTAL PROCEDURES}

The starting material in the present investigation was rolled molybdenum alloy sheet, supplied in the warm worked condition. The sheet provided by Climax Molybdenum contained $0.458 \mathrm{Ti}, 0.68 \mathrm{Zr}, 0.018 \mathrm{~W}$ and $0.0128 \mathrm{C}$. For the major part of the study, the initial thickness of the sheet was $0.5 \mathrm{~mm}$, for the larger recrystallised grain sizes; $1.5 \mathrm{~mm}$ thick sheet obtained from the same batch was used.

The samples were recrystallised in a resistance heated molybdenum furnace. A dynamic vacuum of better than $5 \times 10^{-6}$ torr was ensured during the heating. While under vacuum the 
furnace was brought up to temperature and the specimens were held at temperature for one hour and furnace cooled. The temperatures employed and the resulting grain sizes are listed in Table $I$.

\section{TABLE I}

Description of the Materials Used

\begin{tabular}{ccc}
\hline $\begin{array}{c}\text { Sample } \\
\text { Notation }\end{array}$ & $\begin{array}{c}\text { Annealing } \\
\text { Temperature (K) }\end{array}$ & Grain Size (mm) \\
\hline A & as received & deformed structure \\
B & 1748 & 0.028 \\
C & 1903 & 0.045 \\
D & 2023 & 0.177 \\
E & 2123 & 0.259 \\
\hline
\end{tabular}

Sheet samples $12.7 \mathrm{~mm} \times 12.7 \mathrm{~mm}$ (half inch squares) with the rolling direction inscribed on each were heat treated and glued together to form a cubic stack. Texture measurements were made on a modified Mark VI four circle neutron diffractometer linked to the DIDO reactor at Harwell. Similar experimental methods have been described elsewhere. ${ }^{8}$ Complete (200) and (222) pole figures for the warm worked and recrystallised materials were obtained using this technique. Computer methods were employed to generate the three dimensional biaxial pole figures using William's method ${ }^{9}$ which specifies the volume density of materials for 1296 different crystallite orientations. Symmetry operations were also applied to extend the solution to equivalent orientations present by virtue of the orthotropic character of the texture.

For the determination of anisotropy of elastic properties, it was necessary to apply the stress in a number of orientations with respect to the principal directions of the sheet. Test samples were cut from seven directions in the plane of the sheet at intervals of 15 degrees from the rolling direction to the transverse direction. Resistance strain gauges, $25 \mathrm{~mm} \times 5 \mathrm{~mm}$, were attached to both sides of the specimens. All tests were carried out on an Instron testing machine using a digital strain indicator calibrated to a strain sensitivity of $5 \times 10^{-7}$. Load-unload tests were made in duplicate using small increments of load. The elastic region was characterised by constant readings of strain on unloading. The loading stress at which a permanent strain offset, normally $1 \mathrm{x}$ $10^{-6}$, was first observed on unloading was taken to be the microyield stress. Aspects of macroyielding and plastic flow which were studied ${ }^{10}$ with the same specimens are also to be published. 


\section{RESULTS AND DISCUSSION}

\section{Preferred Orientations}

The predominant texture in the as-received material, as shown in Figure 1, has (100) parallel to the rolling plane and
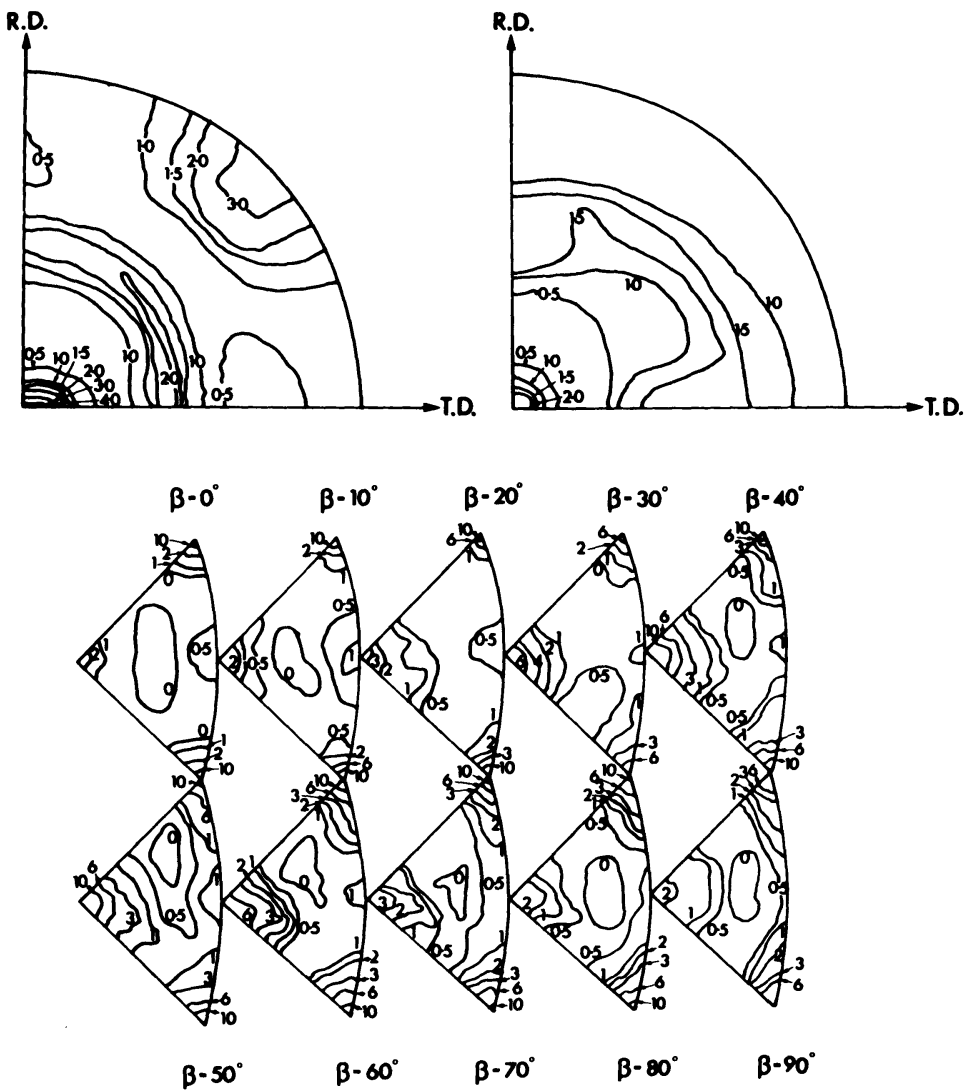

Figure 1. Texture of the warm worked material A. (a) (200) pole figure, (b) (222) pole figure, (c) biaxial pole figure.

[011] along the rolling direction, exhibiting a spread of $17^{\circ}$ about the rolling direction and $7^{\circ}$ about the transverse direction at the $1 \mathrm{x}$ random level. This component of texture manifests itself as an intensity maximum in the (100) corner at $\beta=45^{\circ}$ in the biaxial pole figure, Figure $1(\mathrm{c})$. The other major component is the $\langle 111\rangle / / \mathrm{N}$.D. fibre texture which can be identified as a tube of high intensity centred on the (111) corner in the biaxial volume.

The textures of all the recrystallised materials closely resembled one another. The $(200)$ pole figure for Material $B$ shown in Figure 2 (a) has maxima along the equator and on the circumference along the rolling direction. From the accompanying biaxial pole figure, Figure $2(\mathrm{c})$, the major texture component is $(310)$ [001] which occurs at the (310) pole with 

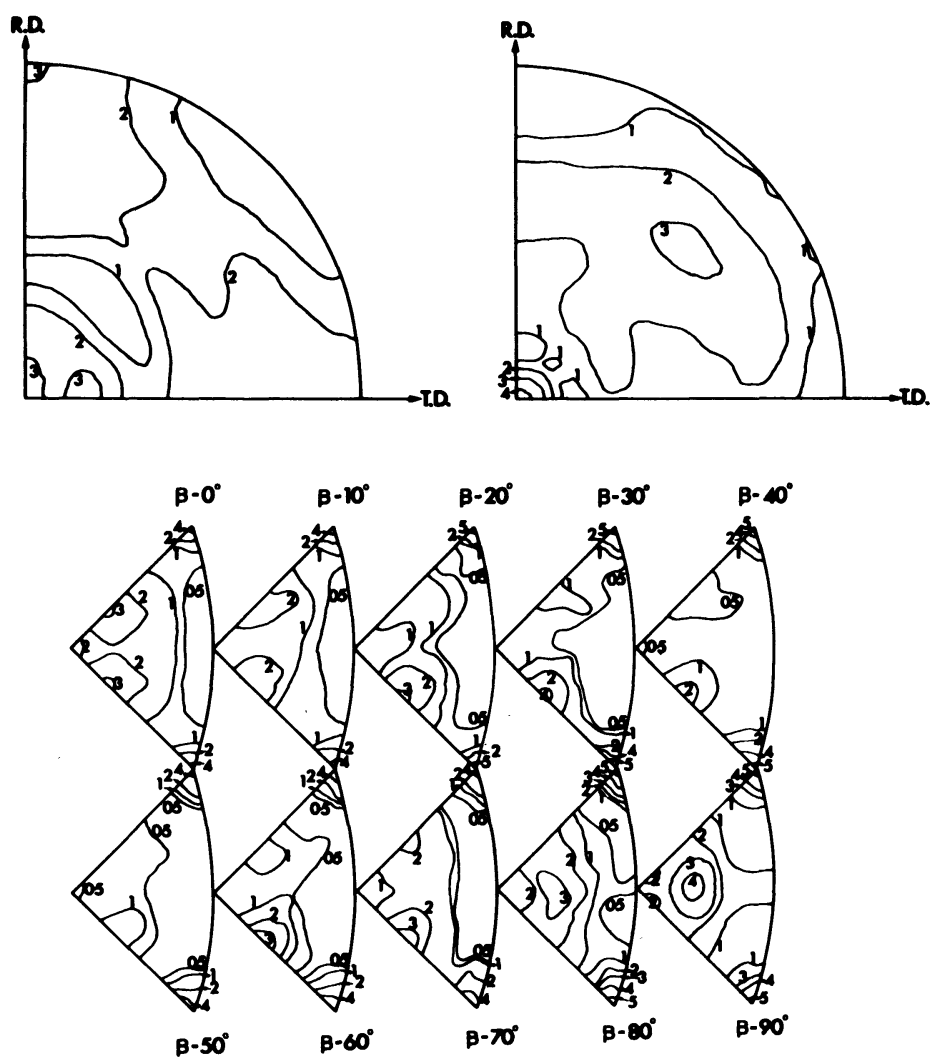

Figure 2. Texture of recrystallised material B. (a) (200) pole figure, (b) (222) pole figure, (c) biaxial pole figure.

$\beta=90^{\circ}$. There is an associated spread in orientations from $(100)$ [001] to $(210)$ [001] and an accompanying <111>//N.D.

fibre texture. The peak positions at the (1Il) corners occur at $\beta=30^{\circ}$ and $\beta=90^{\circ}$ indicating that the (1I1) [112] component is marginally stronger than the (111) [110] orientation.

\section{Elastic Behaviour}

The measured elastic modulus for the as-received material exhibits anisotropic behaviour with a total variation of 58 for testing directions in the plane of the sheet (Figure 3). The Young's modulus is maximum at $45^{\circ}$ from the rolling direction and minimum along the rolling and transverse directions. These trends are consistent with the $\langle 001\rangle$ direction being the elastically hardest orientation in molybdenum. In contrast to the starting material, the Young's modulus after annealing, case B, (Figure 4) is maximum along the rolling direction and decreases as the testing direction deviates from it, exhibiting a minimum at $45^{\circ}$ and finally attains a secondary maximum at the transverse direction. For a larger recrystallised grain size, material D, the anisotropy of Young's modulus follows a pattern similar to the previous case with a minimum of $45^{\circ}$ as shown in Figure 5 . The observed anisotropy 


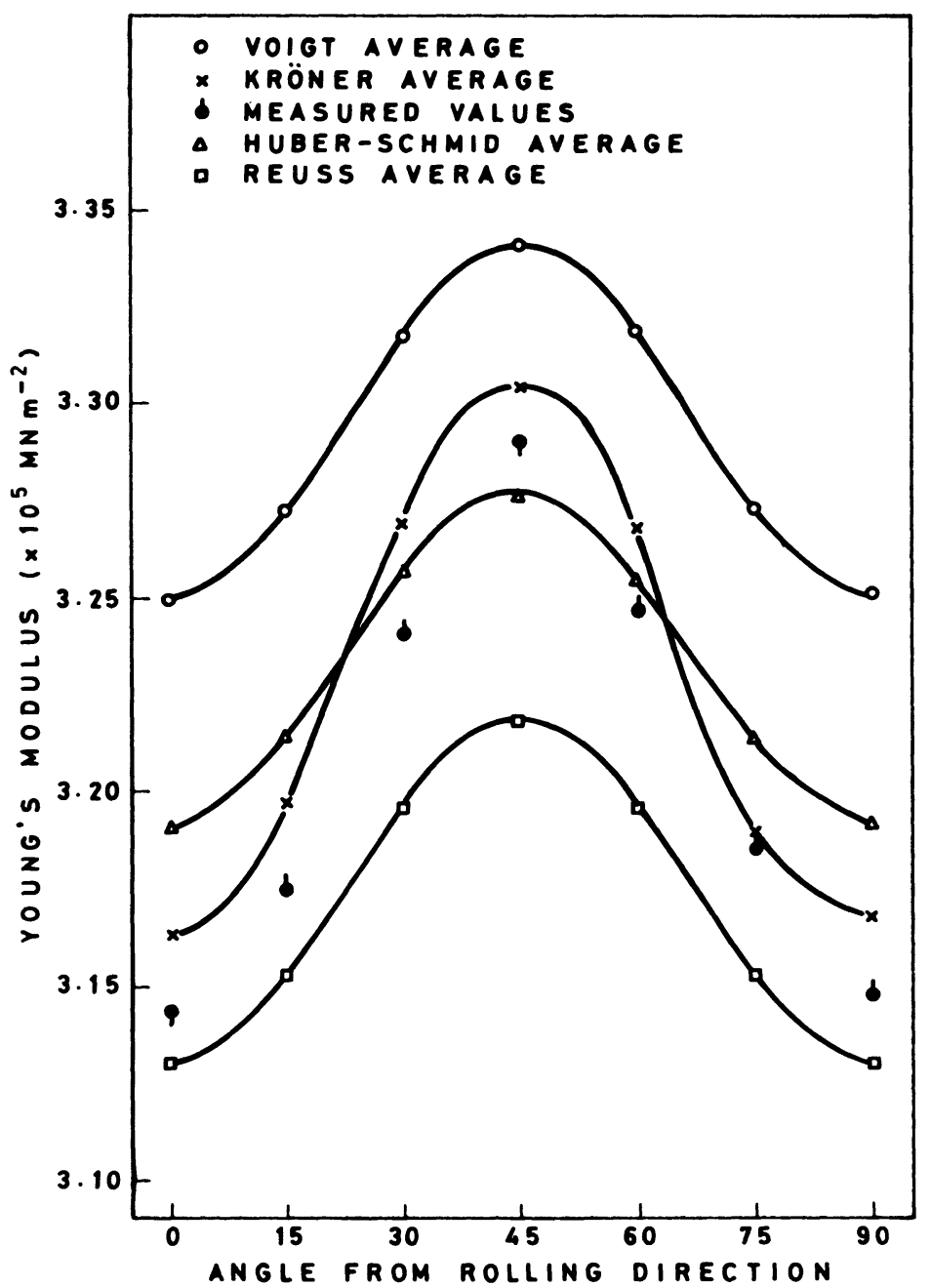

Figure 3. Planar variation of measured and calculated Young's moduli for material $A$.

of Young's modulus is 48 which is lower than that in the worked material. Comparison of Figures 4 and 5 shows that the elastic modulus is remarkably insensitive to grain size.

Models due to Voigt and Reuss have been used to calculate the upper and lower bounds of elastic moduli. The elastic properties of the polycrystalline aggregates are obtained by using the preferred orientation distribution data $V(\Omega)$ and values of the single crystal elastic constants for molybdenum ${ }^{11}$

$$
\begin{aligned}
& \left(c_{11}=453.8, c_{12}=154.7, c_{44}=107.0 \mathrm{GN} / \mathrm{m}^{2}\right) \\
& C_{\text {Voigt }}=\frac{1}{V} S c_{i j k 1}(\Omega) \mathrm{dV} \\
& C_{\text {Reuss }}^{-1}=S_{\text {Reuss }}=\frac{1}{V} \int s_{i j k l}(\Omega) \mathrm{dV}
\end{aligned}
$$




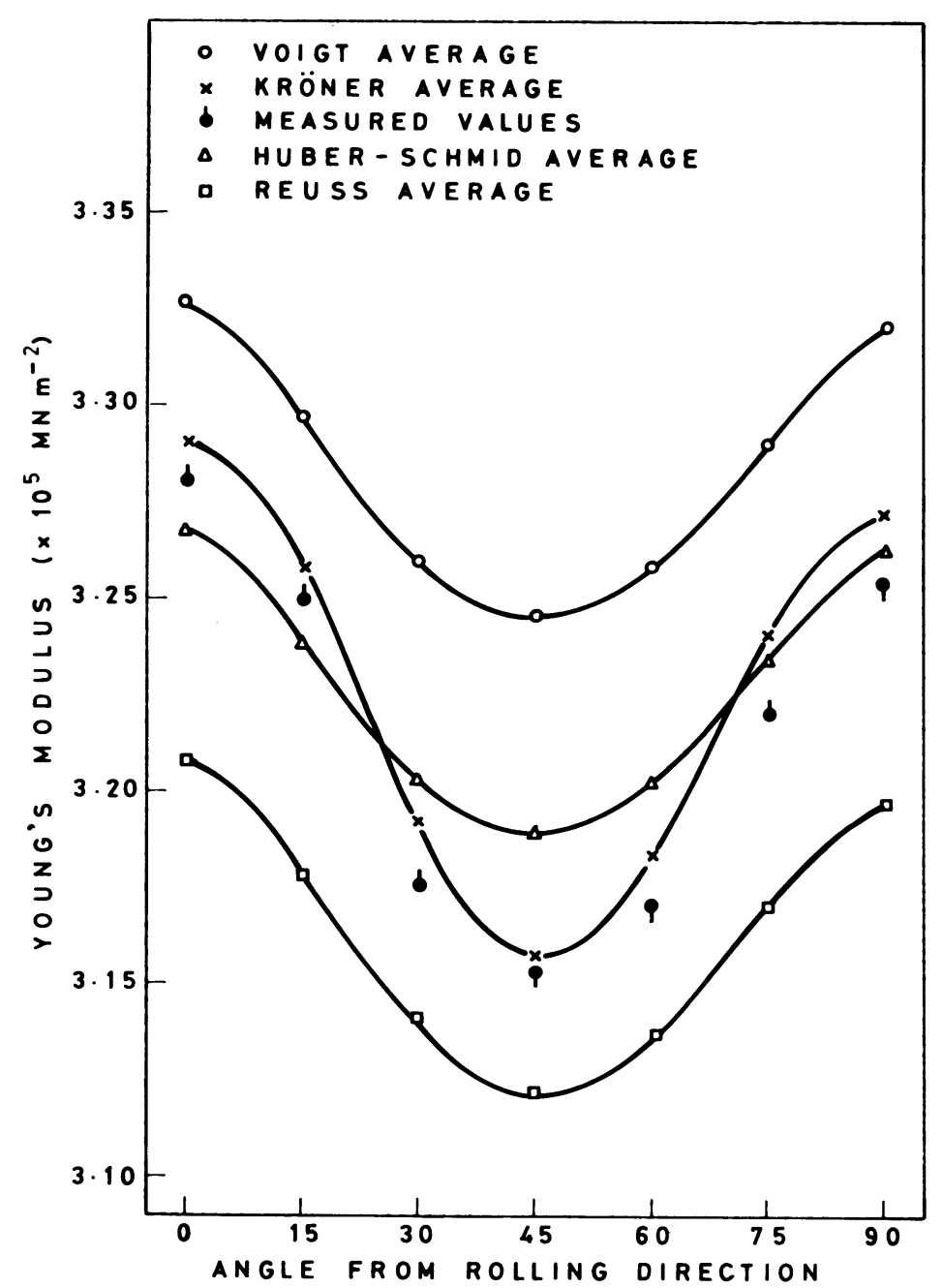

Figure 4. Planar variation of measured and calculated Young's moduli for material $B$.

Calculations of Young's moduli were also made on the basis of the Huber-Schmid method ${ }^{2}$ which assumes that the extensions of all grains are equal along the stress axis. This is simply a space average of the individual crystallite Young's moduli.

The calculated values are shown in Figures 3,4 and 5 for comparison with the experimental results. Curves obtained from the Voigt and Reuss averages lie above and below the measured values, respectively, as would be expected. While the general form of predicted behaviour agrees reasonably well in each case, the degree of variation is greater for the measured values than for the calculated ones.

In the EKK approach, the nonhomogeneous environment of an anisotropic grain in a polycrystal is represented statistically by a homogeneous medium having the macroscopic elastic properties of the anisotropic medium, C. Each grain of orientation $\Omega$ in the polycrystal, with elastic properties $c(\Omega)$, 


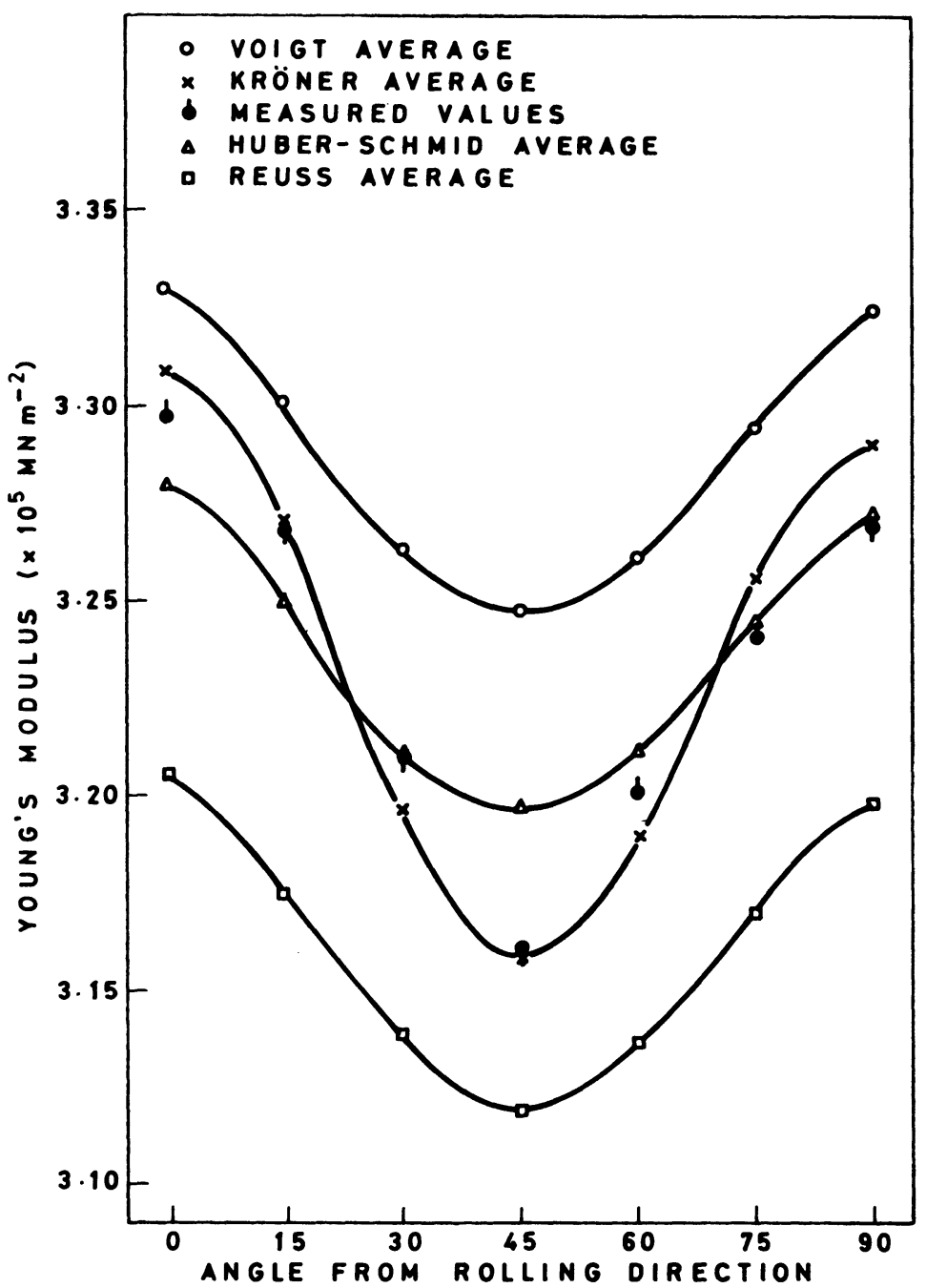

Figure 5. Planar variation of measured and calculated Young's moduli for material $D$.

is considered to be embedded in a medium with the unknown elastic moduli, C. The elasticity problem then requires a solution for $C$ such that the average elastic polarisation in the medium vanishes.

$$
\overline{\mathrm{u}}=\frac{1}{\mathrm{v}} \int \mathrm{u}(\Omega) \mathrm{dv}
$$

where $u(\Omega)=[c(\Omega)-c+c \ldots w]^{-1} \ldots[c(\Omega)-c]$

Components of $w_{i j k l}$ for orthotropic symmetry as in the case of rolled sheet, are defined in terms of $c_{i j k l}$ and $c_{i j k l}$ by Morris. ${ }^{7}$ An initial estimate of $C_{i j k l}$ may be provided by the Voigt or Reuss bounds or an intermediate approximation. The polarisation tensor $\overline{\mathrm{u}}_{i j k l}$ and the polycrystalline elasticity tensor $C_{i j k l}$ have the same symmetry. For the present 
orthotropic case the nine independent components of $\overline{\mathrm{u}}$ are functions of the nine elements of $C$. By sequentially incrementing the elements $C$ in Equation (10), it is possible to define the partial derivatives $U$ :

$$
U_{i j}=\frac{\partial \bar{u}}{\partial C} i, j=1 \text { to } 9
$$

Using a subroutine which minimises a general function of many variables with known first derivatives it has been possible to arrive at a solution of $C$ ( $C^{E K K}$ ) such that the polarisation is very small. In practice a limiting condition

$$
\frac{\left|\bar{u}_{i j k l}\right|}{C_{i j k l}}<10^{-5}
$$

was defined for an acceptable solution. The problem successfully converges to a unique solution very rapidly, independent of the initial value of $C_{i j k l}$. The value of the bulk modulus, $K$, is also preserved at all stages of the calculation. The present approach appears to be more direct than that used by Morris $^{7}$ and readily yields much smaller values of residual polarisation than were quoted for the case of copper. Plots of the planar anisotropy of Young's modulus are obtained for the as-received and recrystallised materials, B and D, by transformation of the CEKK tensor (Figure 3,4 and 5). The compatability analysis gives an excellent match with the measured values of Young's moduli both with regard to the absolute values and the degree of anisotropy which is greater than for the Reuss and Voigt bounds. Implicit in the formulation of the problem, is the fact that the predicted moduli have no dependence on the inclusion (grain) size but depend only on the volume fraction and the orientation present. This behaviour is also borne out by observation.

\section{Microyielding}

Under an externally applied stress, the individual grains in a polycrystalline aggregate deform elastically in a constrained manner until at a critical strain, yielding occurs in isolated grains. The external stress at which this event occurs is designated as the microyield stress. The variation of the microyield stress with angle of testing shown for materials $B$ and $D$ in Figure 6 exhibits similarity in characteristics to the variation of the Young's modulus of the recrystallised materials. Maxima at the rolling and transverse directions and a minimum at $45^{\circ}$ provide a total variation in the microyield stress amounting to 7.38 for material $B$. For the larger recrystallised grain size, $D$, the total variation is 7.08 but in this case the microyield stress levels are reduced by $\sim 1 \mathrm{MN} / \mathrm{m}^{2}$.

The observed behaviour of the microyield stress suggests a strong dependence on the elastic behaviour. Differences in local stress and strain arising from the elastic anisotropy of the aggregate would appear to influence the microyield behaviour. Appropriately, the EKK analysis can be used to 


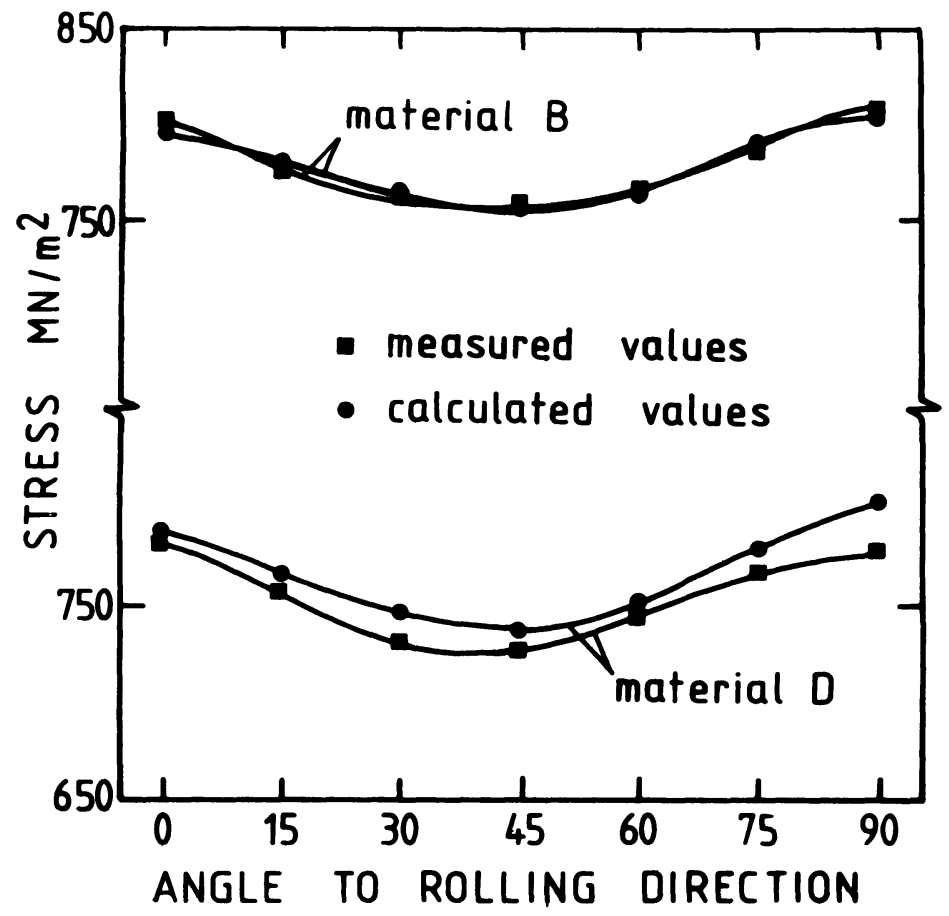

Figure 6. Planar variation of microyield stress (permanent strain $10^{-6}$ ) for materials $B$ and $D$.

calculate the local strain variations under an externally applied stress. Within an inclusion, whose orientation relative to the specimen fixed co-ordinate system is denoted by $\Omega$ the strains $e_{i j}$ caused by the application of a macrostress $\bar{\sigma}_{\mathrm{mn}}$ are given by

$$
e_{i j}(\Omega)=\left[I+u_{i j k l}(\Omega)\right] s_{k 1 m n} \bar{\sigma}_{m n}
$$

The polycrystalline compliances $S_{k} l m n$ are referred to the specimen fixed co-ordinate system. Strains within the inclusion are transformed to the crystal fixed cubic co-ordinate system and are denoted by $e_{k l}^{*}$

$$
e_{k l}^{*}=a_{k i} a_{1 j} e_{i j}
$$

The stresses referred to the cubic system become

$$
\sigma_{\mathrm{mn}}^{*}=c_{\mathrm{mnkl}} \mathrm{e}_{\mathrm{k} 1}^{*}
$$

and can be resolved on each of the twelve possible $\{110\}<111>$ slip systems. For each condition of a uniaxial stress applied in the plane of the sheet, the highest fraction of this resolved stress in the most favourably oriented grain is calculated. The resolved stress for a unit applied stress around the sheet is given in Table II for materials $B$ and $D$. The externally measured microyield stress must vary inversely with the fraction of resolved stress given in Table II. 
TABLE II

Maximum Resolved Shear Stress on the $\{110\}<111\rangle$ Slip for a Unit Applied Tensile Stress

\begin{tabular}{rccc}
\hline \hline Angle to RD & Isotropic Case & Material B & Material D \\
\hline 0 & 0.5 & 0.553 & 0.550 \\
15 & 0.5 & 0.566 & 0.566 \\
30 & 0.5 & 0.577 & 0.580 \\
45 & 0.5 & 0.584 & 0.588 \\
60 & 0.5 & 0.579 & 0.579 \\
75 & 0.5 & 0.557 & 0.557 \\
90 & 0.5 & 0.545 & 0.556
\end{tabular}

Using a value for the resolved shear stress, $\tau_{0}$, of $45 \mathrm{MN} / \mathrm{m}^{2}$ which is in accord with measurements on single crystals at low strains, 13 the anisctropy of the microyield stress was calculated. These results are shown in Figure 6 and demonstrate good agreement between measured and calculated values. A significant conclusion of these observations is that without invoking effects arising from elastic anisotropy, it is not possible to explain the directionality of microyield behaviour since some grains of suitable orientation will always be present. The above analysis highlights the fact that in a moderately anisotropic material like molybdenum, the effect of the elastic anisotropy of the aggregate can raise the maximum resolved stress from 0.5 to 0.6 of the applied stress. Effects arising in highly anisotropic materials such as copper and niobium, previously ignored, must assume considerable significance in the microyielding behaviour of these materials.

\section{CONCLUSIONS}

The elastic moduli of polycrystalline metals can be calculated with considerable accuracy on the basis of the Eshelby-Kröner-Kneer approach. By incorporating measured crystallite orientation distributions the anisotropy of elastic properties has been successfully predicted using this method. The variation in stress from grain to grain in the aggregate resulting from elastic anisotropy is of importance in defining the initiation of plastic flow. Variations in grain size were not found to have a major influence on elasticity or microyielding which is in agreement with the theoretical analysis. 


\section{ACKNOWLEDGEMENTS}

The authors are grateful to UKAEA Harwell for financial support of this work. Laboratory facilities were provided by Professor R. E. Smallman at the University of Birmingham.

\section{REFERENCES}

1. W. Voigt, Lehrbuch der Krystallphysik, Teubner, Berlin, 1910.

2. A. Reuss, Z. Angnew. Math. Mech., 9, 49 (1929).

3. z. Hashin and S. Shtrikman, J. Mech. Phys. Sol., 10, 335 (1962).

4. J. D. Eshelby, Proc. Roy. Soc., A252, 561 (1959).

5. E. Kröner, Z. Phys., 151, 504 (1958).

6. G. Kneer, Phys. Stat. Sol., 9, 825 (1965).

7. P. R. Morris, Int. J. Engr. Sci., 9, 917 (1971)

8. D. E. Stott, Ph.D. Thesis, University of Birmingham, 1975.

9. R. O. Williams, Trans, Met. Soc. AIME, 242, 105 (1968).

10. A. Kumar, Ph.D. Thesis, University of Birmingham, 1975.

11. J. M. Dickinson and P. E. Armstrong, J. Appl. Phys., 38, 602 (1967).

12. A. Huber and E. Schmid, Helv. Phys. Acta, 7, 620 (1934).

13. W. Rinnerthaler and F. Benesovsky, Planseeberichte fur Pulvermetallurgie, 21, 253 (1973). 